\title{
Effects of remote ischemic preconditioning and topical hypothermia in renal ischemia-reperfusion injury in rats ${ }^{1}$
}

\author{
Guilherme Lang Motta', Pablo Cambeses Souza", Emanuel Burck dos Santos'", Silvia Regina Bonalv, \\ Pedro Guilherme Schaeferv, Caetano Araújo Torres Limavı, Norma Anair Possa Marronivil, Carlos \\ Otávio Corso VIII
}

'Fellow Master degree, Postgraduate Program in Medicine: Surgical Sciences, Universidade Federal do Rio Grande do Sul (UFRGS), Porto Alegre-RS, Brazil. Conception and design of the study; acquisition, interpretation and analysis of data; technical procedures; manuscript writing.

"Fellow Master degree, Postgraduate Program in Medicine: Surgical Sciences, UFRGS, Porto Alegre-RS, Brazil. Acquisition and interpretation of data, technical procedures.

"'MSc, PhD, Department of Urology, Hospital de Clínicas de Porto Alegre (HCPA), Porto Alegre-RS, Brazil. Conception and design of the study, critical revision.

IV MSc, PhD, Laboratory of Experimental Gastroenterology and Hepatology, HCPA, Porto Alegre-RS, Brazil. Acquisition and interpretation of data, technical procedures.

${ }^{\vee} \mathrm{MD}$, Department of Pathology, HCPA, Porto Alegre-RS, Brazil. Histopathological examinations, acquisition of data.

${ }^{V I} G$ raduate student, School of Medicine, UFRGS, Porto Alegre-RS, Brazil. Acquisition of data, technical procedures.

VIIFull Professor, Physiologist, Laboratory of Experimental Gastroenterology and Hepatology, HCPA, Porto Alegre-RS, and Laboratory of Oxidative Stress and Antioxidants, Universidade Luterana do Brasil, Canoas-RS, Brazil. Acquisition and interpretation of data, technical procedures.

VIIIAssociate Professor, Department of Surgery, UFRGS, and General Surgery Division, HCPA, Porto Alegre-RS, Brazil. Conception and design of the study, interpretation of data, manuscript writing, critical revision, final approval.

\section{Abstract}

Purpose: To evaluate whether combining hypothermia and remote ischemic preconditioning (RIPC) results in protection from ischemia-reperfusion (IR).

Methods: Thirty-two Wistar rats underwent right nephrectomy and were randomly assigned to four experimental protocols on the left kidney: warm ischemia (group 1), cold ischemia (group 2), RIPC followed by warm ischemia (group 3), and RIPC followed by cold ischemia (group 4). After 240 minutes of reperfusion, histological changes in the left kidney, as well as lipid peroxidation and antioxidant enzyme activity, were analyzed. The right kidney was used as the control. Serum creatinine was collected before and after the procedures.

Results: RIPC combined with hypothermia during IR experiments revealed no differences on interventional groups regarding histological changes $(p=0.722)$. Oxidative stress showed no significant variations among the groups. Lower serum creatinine at the end of the procedure was seen in animals exposed to hypothermia $(p<0.001)$.

Conclusions: Combination of RIPC and local hypothermia provides no renal protection in IR injury. Hypothermia preserves renal function during ischemic events. Furthermore, RIPC followed by warm IR did not show benefits compared to warm IR alone or controls in our experimental protocol.

Key words: Ischemia. Reperfusion Injury. Hypothermia. Free Radicals. Oxidative Stress. Rats. 


\section{Introduction}

Interruption of blood flow through ischemia leads to a cascade of events culminating in cell death. During anaerobic metabolism of ischemic tissues, intracellular hypoxanthine accumulates due to adenosine triphosphate (ATP) degradation. The reintroduction of blood flow through reperfusion paradoxically produces additional damage, which is a phenomenon known as ischemia-reperfusion injury (IR $)^{1}$. This occurs by combining oxygen from tissue reperfusion with hypoxanthine resulting in free radical production, which is extremely harmful to the cellular environment ${ }^{2,3}$. Clinical situations in which we find IR are diverse, such as transplants, extracorporeal circulation, and partial nephrectomies ${ }^{4-6}$.

Several strategies to reduce IR damage have been developed, including the use of hypothermia, hypertonic solutions, antioxidants, and, more recently, ischemic preconditioning (IPC) ${ }^{6}$. Hypothermia is routinely used during organ transplantation preservation and renal surgery by arterial clamping, and is widely used to prevent damage from $\mathbb{I}^{7}$.

Inducing endogenous production of antioxidants through short cycles of ischemiareperfusion prior to prolonged ischemia is the definition of IPC, and is one of the most recent strategies for preventing IR damage ${ }^{8-10}$. The ability to perform IPC in tissues distal to the IR-exposed organ, known as remote ischemic preconditioning (RIPC), makes this a promising and practical transoperative maneuver ${ }^{11,12}$.

There is considerable evidence on the benefits of hypothermia and RIPC as strategies for the prevention of IR damage in contrast to little information on the association of synergistic protective effects. The hypothesis that simultaneous use of cold ischemia and remote preconditioning potentiates its individual effects should be investigated. Therefore, we evaluated the combined effect of RIPC and hypothermia on renal IR injury through a pioneering study with experimental surgery in rats.

\section{- Methods}

The study was approved by the Research Ethics Committee of the Hospital de Clínicas de Porto Alegre (project number, 150471). It is in compliance with the international guidelines of good practices in animal research from the National Institute of Health (Guide for the Care and Use of Laboratory Animals, Bethesda, USA, 2011) and was conducted in accordance with the International Principles for Biomedical Research Involving Animals, published by the Council of International Organizations of Medical Sciences (CIOMS) as well as the Brazilian law on the scientific use of animals (Law 11.794 / 2008).

\section{Animals and study design}

In a prospective design, 32 male Wistar rats weighing between $277-447 \mathrm{~g}$ and aged 54-66 days were randomly divided into four groups (group 1, group 2, group 3, and group 4) of eight animals according to the surgical protocol for the left kidney. Animals were housed in the Animal Experimentation Unit of Hospital de Clínicas de Porto Alegre (HCPA) in groups of four in separate cages at room temperature $\left(22 \pm 2^{\circ} \mathrm{C}\right)$ under a 12-hour light/ dark cycle with free access to water and rat chow.

All 32 animals underwent right nephrectomy at the beginning of the procedure, and 8 of these 32 right kidneys were randomly selected to form the control group (Group 0). In Group 1, ischemia of the left renal pedicle was induced for 40 minutes under euthermia $\left(37^{\circ} \mathrm{C}\right.$; Warm ischemia) followed by reperfusion for 4 hours, when we performed left nephrectomy and euthanasia. In Group 2, left renal pedicle ischemia was performed for 
40 minutes under local hypothermia $\left(4^{\circ} \mathrm{C}\right.$; Cold ischemia) and systemic euthermia followed by reperfusion for 4 hours under euthermia and left nephrectomy. Group 3 underwent RIPC by clamping the left iliac artery for 15 minutes followed by reperfusion for 10 minutes, proceeding to ischemia of the left renal pedicle for 40 minutes under euthermia $\left(37^{\circ} \mathrm{C}\right.$; RIPC + Warm ischemia), new reperfusion for 4 hours, and left kidney withdrawal. Group 4 underwent RIPC by clamping the left iliac artery for 15 minutes followed by reperfusion for 10 minutes under euthermia, proceeding to ischemia of the left renal pedicle for 40 minutes under local hypothermia $\left(4^{\circ} \mathrm{C}\right.$; RIPC + Cold ischemia), and reperfusion for 4 hours under euthermia.

Anesthesia was induced with isoflurane vaporized at $3-5 \%$ by inhalation. Soon after, the animals were placed supine on a heated surgical table; general anesthesia was maintained by vaporization of isoflurane at $2-3 \%$ with an oxygen flow of $1 \mathrm{~L} / \mathrm{min}$, with the reflexes of the right hind paw being periodically tested in order to guarantee adequate anesthesia. The systemic temperature of the animals was constantly measured through a rectal electronic thermometer (Termomed 1.0, Intoterm, Porto Alegre, Brazil). When necessary, a heating lamp was used to maintain the systemic temperature between $35.0^{\circ} \mathrm{C}$ and $37.1^{\circ} \mathrm{C}$.

After inducing general anesthesia, $1 \mathrm{ml}$ of blood was collected through retro-orbital puncture for the initial analysis of creatinine under ophthalmic anesthesia. Then, abdominal trichotomy, intraperitoneal administration of tramadol (10 mg/kg), $0.5 \%$ bupivacaine (1 $\mathrm{mg} / \mathrm{kg}$ ) injection at the median incision site, and, finally, longitudinal median laparotomy were performed. Right total nephrectomy was performed initially after laparotomy in all 32 animals. Half of the kidneys were stored in formalin $10 \%$ for future histopathological analysis; the other half were kept at a temperature of $-80^{\circ} \mathrm{C}$ for analysis of oxidative stress markers.
The left kidney of the animals was submitted to the four experimental nephrectomy protocols mentioned previously. The local temperature in the kidney was maintained under euthermia in groups 1 and 3 , whereas renal hypothermia was applied in groups 2 and 4 during renal pedicle ischemia. This local hypothermia was performed by keeping the kidney in contact with frozen and crushed $0.9 \%$ saline solution and isolated from the other tissues through a fenestrated protective latex tissue; the temperature was controlled at $4^{\circ} \mathrm{C}$ using an intraparenchymal thermometer (BAT 12, IITC Life Science, W. Hills, CA, USA) installed in the renal cortex.

RIPC was induced in groups 3 and 4 by clamping the left common iliac artery under euthermia for 15 minutes and reperfusion for 10 minutes before proceeding to left kidney IR. Left renal pedicle ischemia was performed in all groups (under euthermia in groups 1 and 3 and hypothermia in groups 2 and 4) by clamping the renal pedicle for 40 minutes, followed by reperfusion under euthermia for 240 minutes. Microvascular clamps (Medicon, Tuttlingen, Germany) were used to induce vascular ischemia of the left common iliac artery and left renal pedicle. At the end of 40 minutes of renal ischemia, the vascular clamp was removed and the abdominal wall was sutured. After this, the animal was kept awake for 240 minutes under analgesia (new dose of tramadol $10 \mathrm{mg} / \mathrm{kg}$ ) with free access to water in a heated cage. At the end of the reperfusion period, the anesthetic process and laparotomy were repeated to perform a left nephrectomy. As in the right kidney, half of the left kidneys were kept in formalin $10 \%$ for histopathological evaluation; the other half were stored at a temperature of $-80^{\circ} \mathrm{C}$ for measurement of oxidative activity. At the end of the procedure, a new blood sample was collected through cardiac puncture followed by euthanasia using cardiectomy. 


\section{Histopathological analysis}

Renal tissue was fixed in $10 \%$ formalin, embedded in paraffin, sliced, and stained by the periodic acid-Schiff (PAS) and hematoxylin and eosin (H\&E) methods. Acute tubular necrosis was measured through the scale described by Jablonski et al. ${ }^{13}$ in 5 degrees: 0 , absence of abnormalities; 1 , mitosis and necrosis in individual cells; 2 , necrosis of the cells adjacent to the proximal convoluted tubules, preserving the neighboring tubules; 3 , necrosis restricted to the distal third of the proximal convoluted tubules and a zone of necrosis extending to the inner cortex; and 4, necrosis of all segments of the proximal convoluted tubules (Figure 1). This analysis was performed by a blinded pathologist.

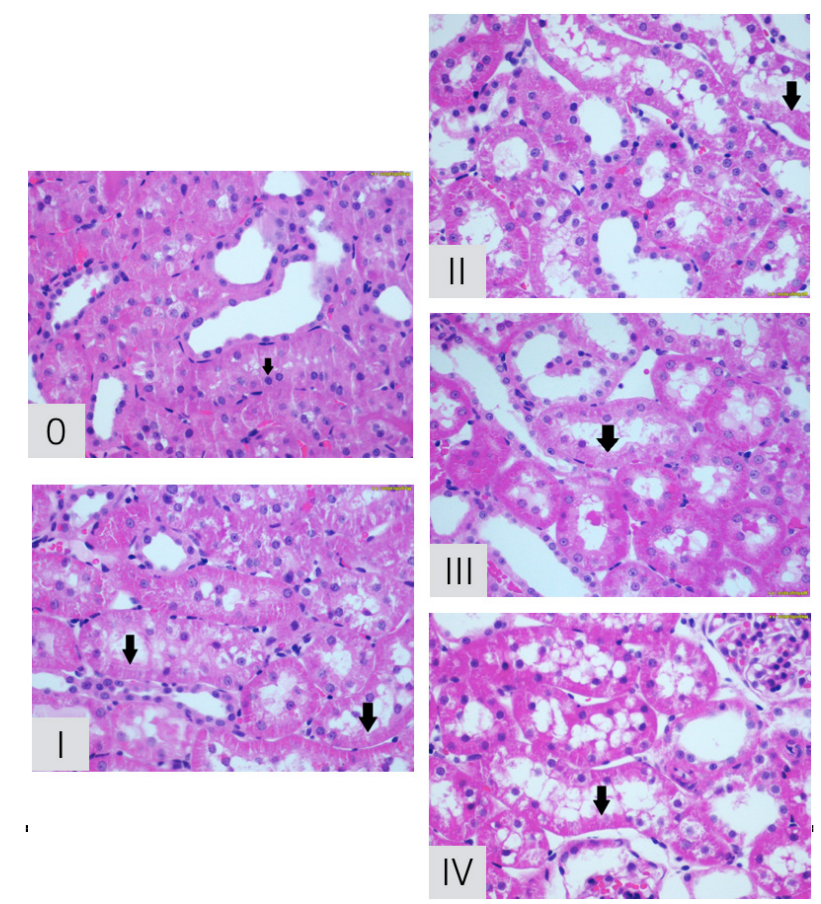

Figure 1 - Acute tubular necrosis degrees by Jablonski. 0 , absence of abnormalities; I, mitosis and necrosis in individual cells; II, necrosis of the cells adjacent to the proximal convoluted tubules, preserving the neighboring tubules; III, necrosis restricted to the distal third of the proximal convoluted tubules and a zone of necrosis extending to the inner cortex; and IV, necrosis of all segments of the proximal convoluted tubules.

\section{Oxidative stress and antioxidant enzyme activity}

Free radicals are biochemically unstable in vivo, with a costly dosage and highly complex practical usage. Thus, lipid peroxidation derivatives are usually quantified ${ }^{14}$. As a renal oxidative stress measure, tissue levels of F2isoprostanes (F2IP), superoxide dismutase (SOD), and catalase (CAT) activities were quantified.

In order to complete the analyses, half of the kidneys preserved at $-80^{\circ} \mathrm{C}$ underwent homogenization for 40 seconds at $4^{\circ} \mathrm{C}$ in the presence of $1.15 \% \mathrm{KCl}(9 \mathrm{~mL} / \mathrm{g}$ tissue) and phenylmethylsulfonyl fluoride at a concentration of $100 \mathrm{mM}$ isopropanol $(10 \mu \mathrm{L}$ per $\mathrm{ml}$ of $\mathrm{KCl}$ ) using Ultra-turrax homogenizer (IKA Works Inc., Wilmington, DE, USA). This homogenate was then centrifuged in a Sorvall Super T21 refrigerated centrifuge (Kendro Laboratory Products, Weaverville, NC, USA), and the supernatant was collected and frozen again at $-80^{\circ} \mathrm{C}$ for biochemical analyses. In addition, protein concentration was measured by the Bradford method using standard bovine albumin and spectrophotometry at $595 \mathrm{~nm}$.

F2-isoprostane (F2IP) is considered a reliable tissue marker of lipid peroxidation, presenting very stable and specific values in the quantification of oxidative stress ${ }^{14}$. Tissue levels of F2IP were determined through the 8-iso-PGF2 $\alpha$ ELISA kit (ADI-900-010, Enzo Life Sciences Inc., Farmingdale, NY, USA) after homogenization of the kidney.

The measurement of superoxide dismutase activity (SOD) was performed by inhibiting superoxide radical reaction with epinephrine in a spectrophotometer at $480 \mathrm{~nm}$ and expressed as USOD/min/mg protein.

Quantification of catalase activity (CAT) was based on the decomposition of hydrogen peroxide in a spectrophotometer at $240 \mathrm{~nm}$ and expressed as pmol/mg protein. 


\section{Biochemical analysis of renal function}

Levels of serum creatinine were determined at baseline from $1 \mathrm{~mL}$ of blood collected through retro-orbital puncture and at euthanasia from blood collected through cardiac puncture using a Roche Cobas 8000 c702 automatic biochemistry apparatus.

\section{Statistical analysis}

Data analysis was performed using a general linear model for statistical significance using SPSS program (SPSS Statistics for Windows, Version 21.0, IBM Corp., Armonk,
NY, USA). Since histological grade is an ordinal variable, Kruskal-Wallis test in conjunction with Dunn's test were used. Bonferroni correction was applied for multiple comparisons between groups. The level of significance was set at 5\% $(p<0.05)$, and data were expressed as mean and standard error (SE).

\section{- Results}

Weight, age, systemic final temperature, and baseline creatinine did not differ between groups of animals (Table 1 ).

Table 1 - Animal weight, age, final systemic temperature, kidney tissue and serum creatinine mean values according to interventional groups

\begin{tabular}{|c|c|c|c|c|c|c|c|c|c|c|}
\hline & ID $^{A}$ & Weight $^{\mathrm{B}}$ & $\mathrm{Age}^{\mathrm{c}}$ & Temp. ${ }^{\mathrm{D}}$ & Histopat. $^{\mathrm{E}}$ & SOD $^{\mathrm{F}}$ & CAT $^{\mathrm{G}}$ & F2IPH & $\begin{array}{c}\mathrm{Cr} \\
\text { (Initial)' }^{\prime}\end{array}$ & $\begin{array}{c}\mathrm{Cr} \\
\text { (Final) }^{\mathrm{J}}\end{array}$ \\
\hline Group 0 & Control & 364.3 & 57 & 36.8 & $0^{*}$ & 7.07 & 0.53 & 26.3 & 0.34 & 1.4 \\
\hline Group 1 & Warm IR & 364.5 & 59 & 36.5 & $2.25(1-4)$ & 6.3 & 0.49 & 25.5 & 0.35 & 1.7 \\
\hline Group 2 & Cold IR & 335.7 & 59 & 36.4 & $2.37(1-4)$ & 8.7 & 0.54 & 26.4 & 0.32 & $0.95 * *$ \\
\hline Group 3 & $\begin{array}{l}\text { RIPC + } \\
\text { Warm IR }\end{array}$ & 370.5 & 59 & 36.8 & $1.8(1-3)$ & 7.6 & 0.61 & 29.8 & 0.33 & 1.66 \\
\hline Group 4 & $\begin{array}{l}\text { RIPC + } \\
\text { Cold IR }\end{array}$ & 380 & 59 & 36.7 & $2.37(1-4)$ & 8.74 & 0.49 & 29.4 & 0.31 & $1.26 * *$ \\
\hline
\end{tabular}

$\mathrm{A}=$ kidneys were identified according to the intervention performed; $\mathrm{B}=$ weight of animals in grams; $\mathrm{C}=$ age of animals in days; $\mathrm{D}$ = final systemic temperature measured in Celsius; $\mathrm{E}=$ kidney histopathological assessment based on Jablonski et al grades of acute tubular necrosis; $F=$ kidney tissue levels of SOD in USOD/min $/ \mathrm{mg} ; \mathrm{G}=$ kidney tissue levels of CAT in pmol/mg; $\mathrm{H}=\mathrm{kidney}$ tissue levels of F2IP in pmol/mL; I = serum creatinine basal levels in $\mathrm{mg} / \mathrm{dL} ; \mathrm{J}=$ serum creatinine final levels in $\mathrm{mg} / \mathrm{dL}$. * Group 0 presented a significantly lower histopathological degree than interventional groups $(p<0.05)$. ** Cold ischemia groups (Group 2 and 4$)$ presented lower final serum creatinine levels compared to warm ischemia animals $(p<0.001)$

Histological analysis identified all grades of acute tubular necrosis ranging from 0 to 4 . It was not possible to ascribe a specific grade to an individual interventional group due to overlapping of findings. The control group, however, presented only grade 0 damage, which was not found in any other group.
Furthermore, grade 4 absence occurred only in experimental group 3 (remote preconditioning with warm ischemia) and the controls.

There was a significant difference between groups regarding histological grade and the controls $(p=0.001)$. The control group presented a significantly lower degree 
than all other groups $(p<0.05)$, as shown in Figure 2. Among interventional groups, there was no statistically significant difference $(p=0.722)$.

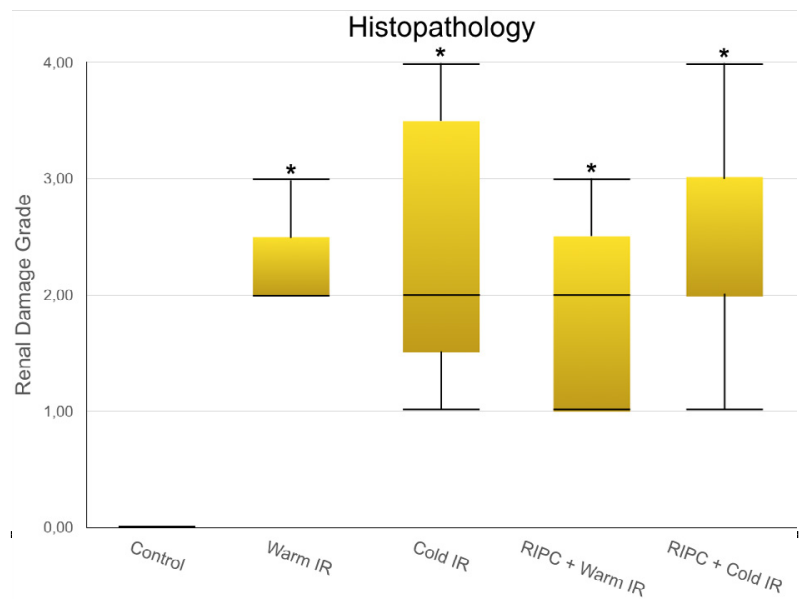

Figure 2 - Comparison between groups and histopathological findings. Control group present lower degree of renal damage $(p<0.05) .{ }^{*}$ Among interventional groups, there is no statistically significant difference $(p=0.722)$. Data are expressed as mean $\pm \mathrm{SE}$. Kruskal-Wallis test complemented by Dunn is used.

Oxidative stress levels were measured by F2IP tissue survey and revealed no statistically difference between interventional groups and control kidneys. When comparing exclusively the warm ischemia groups (Group 1 and 3), we observed that those exposed to RIPC before warm IR presented higher F2IP levels than those in the warm ischemia without preconditioning group $(p<0.05)$ (Figure 3). Catalase measurements did not differ statistically between interventional and control groups $(p=0.736)$ (Figure 4$)$.

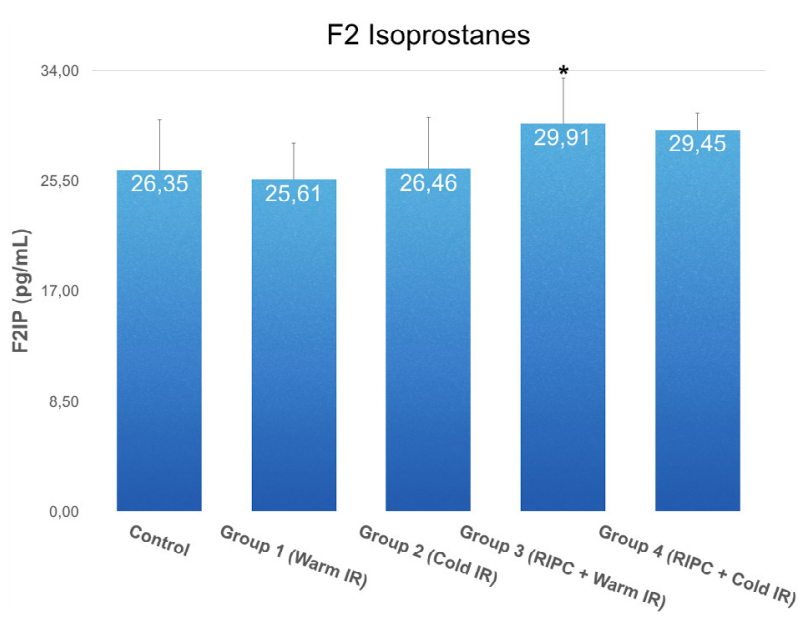

Figure 3 - Comparison between groups and F2isoprostanes tissue levels. No statistically difference between interventional groups and control kidneys. *Among warm ischemia groups, we observe that those submitted to RIPC before warm IR present higher F2IP levels than warm ischemia without preconditioning $(p<0.05)$. Data are expressed as mean $\pm \mathrm{SE}$. Generalized linear models were applied. Bonferroni correction was applied to the significance levels of each test.

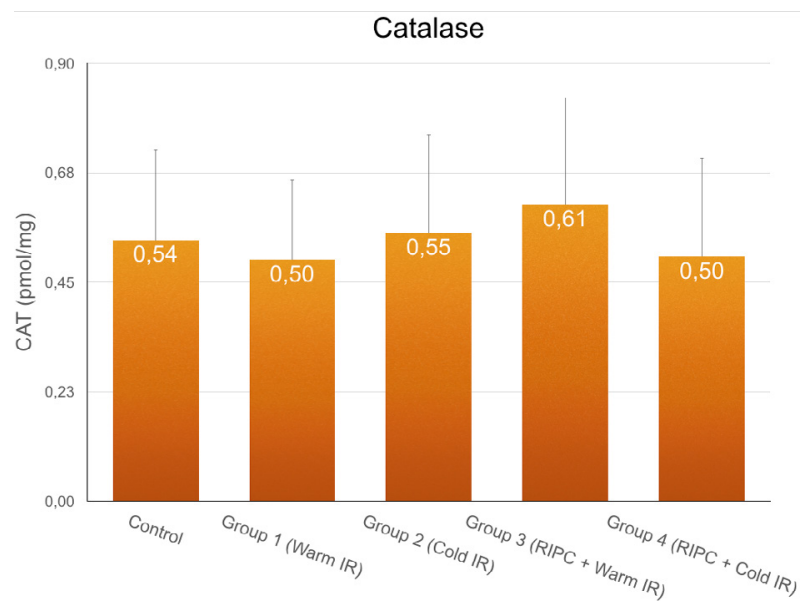

Figure 4 - Comparison between groups and CAT activity. CAT levels do not differ statistically between interventional and control groups $(p=0.736)$. Data are expressed as mean \pm SE. Generalized linear models were applied. Bonferroni correction was applied to the significance levels of each test. 
SOD activity showed higher levels in cold ischemia (groups 2 and 4) compared to that in warm ischemia without RIPC (group 1$)(p<0.05)$; however, there was no difference between the interventional groups and control group $(p=0.736)$ (Figure 5).

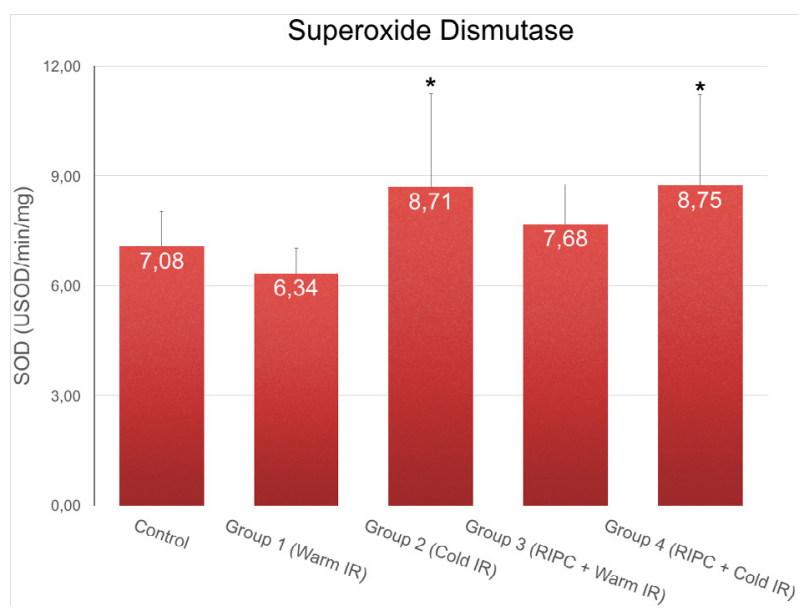

Figure $\mathbf{5}$ - Comparison between groups and SOD activity. *SOD activity show higher levels in cold ischemia (groups 2 and 4) compared to warm ischemia without RIPC (group 1) $(p<0.05)$, however there is no difference between experimental groups and control. Data are expressed as mean \pm SE. Generalized linear models were applied. Bonferroni correction was applied to the significance levels of each test.

Biochemical analysis of renal function at the end of the procedure revealed lower serum creatinine levels in cold ischemia groups (with or without RIPC) compared to the warm ischemia sample (with or without RIPC) $(p<$ 0.001) (Table 1).

\section{- Discussion}

Ischemic preconditioning (IPC) is a maneuver in which short periods of ischemia and reperfusion precede prolonged ischemia and subsequent reperfusion, promoting a conditioning stimulus at the target organ (local preconditioning [LIPC]) or at distant tissues (remote preconditioning [RIPC]). This is an effective strategy in reducing injury through the tissue IR process ${ }^{4,6,8}$.

The first analysis on the effects of preconditioning was described by Murry et al. ${ }^{8}$ in 1986 where the effects of LIPC on canine myocardium were evaluated. Since then, several studies involving different animals, organs, tissues, and IPC protocols have been described.

The effects of preconditioning on renal tissue were first described in 1997, when Islam et al. ${ }^{9}$ performed LIPC in rat kidneys. The use of protocols based on myocardial experiments revealed discouraging results. However, this study identified that the ischemia period should be more than 20 minutes when there is intent to promote renal injury; further, a duration of 40 minutes was found to be ideal for this purpose.

In 2002, Torras et al. ${ }^{6}$ performed a complex study using different variations of IPC in the kidneys of rats, reaching the current bases of preconditioning protocols. In study occasion, it was evidenced that LIPC was shown to be more nephroprotective when performed with a single 15-min cycle of ischemia followed by reperfusion for $10 \mathrm{~min}$ before subsequent prolonged IR. Due to the similarity with our study, this was the protocol used during our experiments.

An alternative to $\mathrm{PCl}$ is RIPC, which is defined as short cycles of ischemia-reperfusion in a tissue distant from another which will undergo a prolonged cycle of IR. The concept of RIPC arose in 1993 when a canine experimental study by Przyklenk et al. ${ }^{15}$ showed that inducing short IR cycles in a coronary vascular territory caused myocardial protection of another vascular territory during subsequent cardiac IR. Gho et al. ${ }^{16}$ expanded the potential of RIPC by reporting benefits of short cycles of ischemia in the mesenteric artery prior to myocardial IR in 1996, validating the hypothesis that tissues used for IPC do not need to be from the same organ under IR. 
The first study involving RIPC and nephroprotection occurred in 2002, when Ates et al. ${ }^{17}$ performed a short cycle of hepatic ischemia and reperfusion prior to sustained renal ischemia in rats. Animals submitted to this protocol presented better biochemical and histopathological renal parameters when compared to rats exposed exclusively to IR without preconditioning. Wever et al. ${ }^{18}$ were pioneers in studying renal IR by inducing RIPC through tourniquets in the hind limbs of rats in 2011, similarly to our study protocol. At that time, different RIPC protocols were tested, with nephroprotection occurring in all groups when compared to IR without RIPC. It is interesting to mention that there was a superiority of bilateral protocols over unilateral ones and, in addition, protocols of fractional cycles showed discretely better results comparing with those with a single cycle. One of the most important studies on renal IPC is a meta-analysis on 58 experimental articles, which compared several protocols. The results revealed equivalent benefits between local and remote ischemic preconditioning ${ }^{12}$.

The preconditioning mechanism of protection remains to be clarified despite the result of several studies. It is believed that there is an endogenous production of antioxidants that could reduce intracellular oxidative stress. In addition, it is known that the production of autacoids (adenosine, opioids, endothelin, and bradykinin) is involved in this process, since

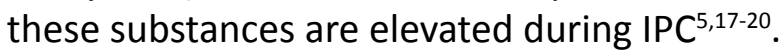

The potential to generate benefits without subjecting the target organ to multiple ischemia and reducing local vascular complications (e.g., arterial thrombosis) is encouraging for the clinical application of PCIR. Its use has been described with reassuring results in the preservation of renal function during procedures associated with renal IR, such as aortic clamping, extracorporeal circulation, renal transplantation, and partial nephrectomy $y^{5,20,21}$.
Hypothermia causes minor cellular metabolism and is another strategy for reducing tissue damage from the IR process, since renal oxidative stress is lower under low temperatures compared to its euthermic pairs ${ }^{22}$. The benefit of local hypothermia in renal tissue varies according to temperature, as demonstrated by Santos et al. ${ }^{22}$ in a study demonstrating that the temperature of $4^{\circ} \mathrm{C}$ leads to a lower oxidative activity in renal tissue submitted to IR in Wistar rats compared to that in other degrees of hypothermia. Fiorentini et al. ${ }^{23}$ also identified that performing local hypothermia during renal IR reduces inflammatory activity in remote organs not involved in the IR (e.g., lung), revealing the systemic benefits of this maneuver.

Other variables can influence the effects of hypothermia, including the tissue preservation solution and perfusion protocols. There are several means of preserving organs for transplantation, such as the University of Wisconsin and Euro-Collins solutions. It seems that the former promotes greater survival of renal grafts during transplantation ${ }^{24}$.

Despite several studies evaluating ischemic preconditioning, there are no studies combining RIPC and hypothermia to identify whether there is a synergistic protective effect when applying these two modalities for protection against the effects of prolonged IR on renal tissue. The association between local renal preconditioning and transoperative hypothermia was investigated by Ribeiro et $a l^{25}$, when LIPC was performed with and without low kidney temperatures. The results did not demonstrate the synergistic effects of LIPC concomitant with hypothermia in renal protection during IR. In fact, hypothermia benefits were reduced when the kidneys were exposed to LIPC.

Considering the feasibility and benefits of RIPC or hypothermia, we performed the present study to elucidate the potential combination of its effects on IR lesions. We 
produced a short-term experiment using histopathological, oxidative stress and creatinine as endpoints.

In our study, only male rats were selected to keep the sample as homogeneous as possible. There is also evidence of a greater susceptibility of this sex to renal injury due to IR. It is believed that estrogen has protective potential for renal tissue under ischemia, a fact that should be better clarified in the future ${ }^{26}$.

Our research group utilizes
experimental models in rodents and has published several studies on renal and hepatic IR in these animals. In this study we followed the routines of our surgery laboratory using rats. Rodent model of experimental surgery is easier and demands a lower cost to manage in laboratory, however there are some limitation. Extrapolation of results to human is limited due to differences in kidney anatomy between these two species. Another disadvantage with rodents is that even a short period of renal artery occlusion leads to extensive necrosis of the proximal tubules. Swine is considered the gold-standard experimental animal model for IR, with surgical and anesthesia protocols very similar to human. Furthermore, pig is one of the few species characterized by a human-like anatomy with multipapillary and multilobular kidneys. Nevertheless, the size of swine limits its use, requiring adapted area, more expenses with food, drugs and surgical materials ${ }^{27}$.

The unique anesthesia protocol and preoperative preparation were performed in all animals, since many substances administered could protect or damage the tissue under ischemia and reperfusion. Tramadol, an opioid analgesic used in our experiment, was recently associated with an influence on ischemic preconditioning, according to Oliveira et al. ${ }^{19}$ This study demonstrated that tramadol reduces renal IR damage when used alone and associated with RIPC. However, there was no positive synergistic effect between RIPC and tramadol, since performing RIPC without administering tramadol has been shown to be more effective in reducing renal damage than that when combined ${ }^{19}$. Another drug used in our study with influence on the IR process is isoflurane, which was used as an inhalation anesthetic. There is evidence of the preconditioning effects of the drug itself and this information should be considered when interpreting the results of our study ${ }^{28}$.

In analyzing the histopathological changes, we observed that there were no differences among the experimental groups, even when comparing single warm IR with hypothermia or preconditioning protocols. However, there was a statistical difference with control kidneys, meaning that this experimental model of surgery is capable of inducing damage.

The absence of benefits from IPC is previously reported in one of the largest meta-analysis of experimental ischemic preconditioning, where 12 of 26 studies included did not demonstrate advantages in performing IPC regarding histological changes ${ }^{12}$. In this meta-analysis, the authors analyzed 205 controls and 191 IR animals submitted to different ischemic preconditioning protocols (including local and RIPC) for a subsequent IR and histopathological assessment. An overall significant IPC protective effect against tissue damage was found; however, a strong trend towards a late window of protection is mentioned, suggesting that these advantages were more pronounced when kidneys were collected at least 24 hours after preconditioning. Furthermore, an overall study heterogeneity of $63 \%$ is also cited by the authors. In our study, left nephrectomies occurred 240 minutes after IPC, which is considered an early window, and this could justify the lack of differences among the interventional protocols in our study.

Despite considerable evidence on renal protection using hypothermia, lack of protection or histologic damage due to cold temperatures is also mentioned ${ }^{22,29}$. In a 
similar experiment using acute reperfusion, Santos et al..$^{22}$ could not prove statistically that hypothermia reduces histological changes in short-time IR. Moreover, another study comparing structural changes in renal rats exposed to a single episode of hypothermia showed that rewarmed kidneys (kidneys exposed to cold and subsequent warm temperatures) have more tubular changes than persistently cold kidneys ${ }^{29}$. Our study used a short-time rewarmed protocol that demonstrated no differences on histology after hypothermic IR. The combination of remote ischemic preconditioning and hypothermia was also neither beneficial nor detrimental, compared to single ischemia-reperfusion.

Oxidative stress measured by F2IP tissue levels demonstrated that euthermic RIPC unexpectedly increased isoprostanes compared to single warm IR, but no differences from controls were observed in this regard. Remote IPC associated with hypothermia resulted in similar F2IP levels compared to those in other interventional protocols and control. Antioxidant enzymes activities defined by CAT and SOD levels also revealed no divergence between RIPC, hypothermia or combined (RIPC + hypothermia) protocols, and controls. Albeit cold protocols induced more antioxidant activity in the SOD analysis, these groups did not differ from controls. This shortage of statistically variation in oxidative stress data probably results from the acute nature of our experiment, with kidneys being collected after only four hours of reperfusion.

Regarding renal function parameters, our study demonstrates that cold protocols (cold IR and RIPC + Cold IR) promote lower serum creatinine levels after IR compared to those in warm protocols. Performing remote preconditioning has a non-statistically significant trend to protect kidneys in warm IR. Nevertheless, the combination of hypothermia and RIPC reduces the benefits of hypothermia alone with no associated synergistic protection.
In summary, our study reveals that RIPC combined with hypothermia did not confer protection against histological, oxidative, or functional changes in kidneys after IR. Additionally, there is no benefit in performing RIPC in this model of experimental surgery. Despite these results, hypothermia protocols demonstrate better functional outcomes than warm IR.

The main strength of this research is that, to the best of our knowledge, this is a pioneer study on the influence of remote ischemic preconditioning on hypothermia during renal damage from IR. Although we did not achieve positive results in this combination, further studies are necessary to validate these experimental models.

The limitations in our study must be acknowledged. Initially, short-term experiments such as ours could preclude the long-term effects of ischemic preconditioning of IR. Besides, the use of rodents in the experiments is considered a limitation due to anatomical differences and that even a short period of renal artery occlusion could lead to extensive tubule necrosis ${ }^{27}$. Furthermore, we assessed the "early window of protection", with evidence that "late" protocols (IPC 24-hours before $(R)$ are more efficient in reducing IR damage ${ }^{12}$. Moreover, even though the final systemic temperatures were not statistically different, we recognize that many animals from cold protocols had some variation in their temperature, which could contribute to bias. Finally, the drugs used during surgery could interfere with our results, since recent data reveal that isoflurane and tramadol could also produce preconditioning effects and confound our outcomes ${ }^{19,28}$.

\section{Conclusion}

No synergistic benefits were found when remote ischemic preconditioning was applied to hypothermia in renal IR. 
Furthermore, hypothermia promotes better functional outcomes than warm protocols.

\section{References}

1. Salvadori M, Rosso G, Bertoni E. Update on ischemia-reperfusion injury in kidney transplantation: pathogenesis and treatment. World J Transplant. 2015;5(2):5267. PMID: 26131407.

2. Bulkley GB. Free radical-mediated reperfusion injury: a selective review. $\mathrm{Br}$ J Cancer Suppl. 1987 Jun;8:66-73. PMID: 3307876.

3. SharfuddinAA, MolitorisBA. Pathophysiology of ischemic acute kidney injury. Nature Rev Nephrol. 2011 Apr 1;7(4):189-200. PMID: 21364518.

4. Schmidt MR, Smerup M, Konstantinov IE, Shimizu M, Li J, Cheung M. Intermit-tent peripheral tissue ischemia during coronary ischemia reduces myocardial in-farction through a KATP-dependent mechanism: first demonstration of remote ischemic preconditioning. Am J Physiol Heart Circ Physiol. 2007 Apr;292(4):1883-90. PMID: 17172279.

5. Ali ZA, Callaghan CJ, Lim E, Ali AA, Nouraei SAR. Remote ischemic precondi-tioning reduces myocardial and renal injury after elective abdominal aortic aneu-rysm repair: a randomized controlled trial. Circulation. 2007; Sep:116(11 Suppl):198-105. PMID: 17846333.

6. Torras J, Herrero-Fresneda I, Lloberas N, Riera M, Ma Cruzado J, Ma Grinyó J. Promising effects of ischemic preconditioning in renal transplantation. Kidney Int. 2002 Jun;61(6):2218-27. PMID: 12028463.

7. Susantitaphong P, Alfayez M, Cohen-Bucay A, Balk EM, Jaber BL. Therapeutic hypothermia and prevention of acute kidney injury: a meta-analysis of random-ized controlled trials. Resuscitation. 2012 Feb;83(2):159-67. PMID: 21983123.

8. Murry $C E$, Jennings RB, Reimer KA. Preconditioning with ischemia: a delay of lethal cell injury in ischemic myocardium. Circulation. 1986 Nov;74(5):1124-36. PMID: 3769170.

9. Islam CF, Mathie RT, Dinneen MD, Kiely EA, Peters AM, Grace PA. Ischaemia- reperfusion injury in the rat kidney: the effect of preconditioning. Br J Urol. 1997 Jun;79(6):842-7. PMID: 9202547.

10.Toosy N, McMorris EL, Grace PA, Mathie RT. Ischaemic preconditioning pro-tects the rat kidney from reperfusion injury. BJU Int. 1999 Sep;84(4):489-94. PMID: 10468768.

11.Przyklenk K, Darling CE, Dickson EW, Whittaker P. Cardioprotection "outside the box"- the evolving paradigm of remote preconditioning. Basic Res Cardiol. 2003;98(3):149-57. PMID: 12883832.

12. Wever KE, Menting TP, Rovers $M$, van der Vliet JÁ, Rongen GA. Ischemic preconditioning in the animal kidney - a systematic review and meta-analysis. PLos ONE. 2012 Feb;7(2):e32296. doi:10.1371/ journal.pone.0032296.

13.Jablonski P, Howden BO, Rae DA, Birrell CS, Marshall VC, Tange J. An exper-imental model for assessment of renal recovery from warm ischemia. Trans-plantation. 1983 Mar;35(3):198-204. PMID: 6340272.

14. Halliwell B, Whiteman M. Measuring reactive species and oxidative damage in vivo and in cell culture: how should you do it and what do the results mean? $\mathrm{Br} J$ Pharmacol. 2004 May;142(2):231-55. PMID: 15155533.

15.Przyklenk K, Bauer B, Ovize M, Kloner RA, Whittaker P. Regional ischemic "preconditioning" protects remote virgin myocardium from subsequent sustained coronary occlusion. Circulation. 1993 Mar;87(3):893-9. PMID: 7680290.

16. Gho BC, Schoemaker RG, van den Doel MA, Duncker DJ, Verdouw PD. Myo-cardial protection by brief ischemia in noncardiac tissue. Circulation. 1996;94(9):2193-200. PMID: 8901671.

17.Ateş E, Genç E, Erkasap N, Erkasap S, Akman S, Firat P, Emre S, Kiper H. Renal protection by brief liver ischemia in rats. Transplantation. 2002 Nov 15;74(9):124751. PMID: 12451261.

18. Wever KE, Warlé MC, Wagener FA, van der Hoorn JW, Masereeuw R, van der Vliet JA, Rongen GA. Remote ischaemic preconditioning by brief hind limb ischaemia protects against renal ischaemiareperfusion injury: the role of adenosine. Nephrol Dial Transplant. 2011 Oct;26(10):3108-17. PMID: 21427077. 
19.Oliveira RC, Brito MV, Ribeiro RF, Oliveira LO, Monteiro AM, Brandão FM, Cavalcante LC, Gouveia EH, Henriques HY. Influence of remote ischemic con-ditioning and tramadol hydrochloride on oxidative stress in kidney ische-mia/reperfusion injury in rats. Acta Cir Bras. 2017 Mar;32(3):229-35. PMID: 28403347.

20.Huang J, Chen Y, Dong B, Kong W, Zhang J, Xue W. Effect of remote is-chaemic preconditioning on renal protection in patients undergoing laparoscopic partial nephrectomy: a blinded randomized controlled trial. BJU Int. 2013 Mar;112(1):7480. PMID: 23452148.

21.MacAllister R, Clayton T, Knight R, Robertson $S$, Nicholas J, Motwani M, Veighey K. Remote preconditioning for protection against ischaemia-reperfusion in renal transplantation (REPAIR): a multicentre, multinational, dou-ble-blind, factorial designed randomised controlled trial. Efficacy Mech Eval. 2015 May;2(3):1-60. PMID: 26020087.

22.Santos EBD, Koff WJ, Grezzana Filho T de JM, De Rossi SD, Treis L, Bona SR, Pêgas KL, Katz B, Meyer FS, Marroni NA, Corso CO. Oxidative stress evaluation of ischemia and reperfusion in kidneys under various degrees of hy-pothermia in rats. Acta Cir Bras. 2013;28(8):568-73. PMID: 23896835.

23. Fiorentini MR, Santos EB, Longo L, Kliemann LM, Koff WJ, Corso CO. Analysis of the effects of topical renal hypothermia on lung tissue after kidney ischemia and reperfusion in rats. Acta Cir. Bras. 2015 Jul; 30(7):445-51. PMID: 26270135.

24.Novick AC. Renal hypothermia: in vivo and ex vivo. Urol Clin North Am. 1983 Nov;10(4):637-44. PMID: 6356550.

25. Ribeiro GBS, Santos EB, Bona SR, Schaefer PG, Garcez TNA, Rabolini EB, Smaniotto GP, Marroni NAP, Corso CO. The effects of local ischemic precondi-tioning and topical hypothermia in renal ischemia/ reperfusion injury in rats. Acta Cir Bras. 2017 Oct;32(10):816-26. PMID: 29160368.

26. Metcalfe PD, Meldrum KK. Sex differences and the role of sex steroids in renal injury. J Urol. 2006 Jul;176(1):15-21. PMID: 16753358.

27.Giraud S, Favreau F, Chatauret N, Thuillier $R$, Maiga $S$, Hauet $T$. Contribution of large pig for renal ischemiareperfusion and transplantation studies: the preclini-cal model. J Biomed Biotechnol. 2011;2011:532127. doi: 10.1155/2011/532127.

28. Menting TP, Ergun $\mathrm{M}$, Bruintjes $\mathrm{MH}$, Wever $\mathrm{KE}$, Lomme RM, van Goor $\mathrm{H}$, War-lé MC. Repeated remote ischemic preconditioning and isoflurane anesthesia in an experimental model of renal ischemia-reperfusion injury. BMC Anesthesiol. 2017 Jan 28;17(1):14. PMID: 28129737.

29.Tveita T, Johansen $\mathrm{K}$, Lien AH, Myklebust R, Lindal S. Morphologic changes in tubular cells from in situ kidneys following experimental hypothermia and re-warming. APMIS. 2005 Jan;113(1):13-20. PMID: 15676010.

\section{Correspondence:}

Carlos Otávio Corso

Disciplina de Cirurgia Geral, Hospital de Clínicas

de Porto Alegre

Rua Ramiro Barcelos, 2350/747

90035-903 Porto Alegre - RS Brasil

corso@portoweb.com.br

Received: Jan 09, 2018

Review: Mar 08, 2018

Accepted: Apr 12, 2018
Conflict of interest: none

Financial source: FIPE 